\title{
Effects of initial molten pool and Marangoni flow on solid melting
}

\author{
X.F. Peng ${ }^{\mathrm{a}, *}$, X.P. Lin ${ }^{\mathrm{a}}$, D.J. Lee ${ }^{\mathrm{b}}$, Y. Yan ${ }^{\mathrm{a}}$, B.X. Wang ${ }^{\mathrm{a}}$ \\ ${ }^{a}$ Thermal Engineering Department, Tsinghua University, Beijing 100084, China \\ ${ }^{\mathrm{b}}$ Chemical Engineering Department, Taiwan University, Taipei 106, Taiwan
}

Received 24 May 1999; received in revised form 8 February 2000

\begin{abstract}
This study investigated the role of initial molten pool on the numerical simulation to melting process. The critical time for heat conduction mechanism to control the formation of initial molten pool is estimated first. A realistic formulation to the initial molten pool is then proposed. The linear stability characteristics of the initial molten pool when subject to Marangoni flow induced by the perturbations of surface liquid temperature were also analyzed. For initial pool having a melting time less than the critical time, the subsequent numerical results are independent of the assumed initial pool. An arbitrarily assumed initial pool could yield erroneous numerical results for melting. Detailed numerical investigations on the shape evolutions of molten pool during initial stage and the long-term stage were conducted. Effects of the heat transfer intensity and the Marangoni number on the melting process were also investigated. (C) 2000 Elsevier Science Ltd. All rights reserved.
\end{abstract}

Keywords: Melting; Initial molten pool; Phase-change interface; Marangoni effect; Numerical simulation; Linear stability

\section{Introduction}

Melting of solid is commonly found in many practical applications like crystal growth and laser processing to metals. Traditional approach, the so-called Stefan problem, assumed a heat conduction-controlled process; that is, there exists no motion of liquid in the molten pool during melting. Till late 1970s the effects of fluid flow to the melting process were recognized. Owing to the incorporation of fluid motion numerical analysis or experimental investigation is necessary to

\footnotetext{
* Corresponding author. Tel.: + 86-10-62789751; fax: + 8610-62770209.

E-mail address: pxf-dte@tsinghua.edu.cn (X.F. Peng).
}

the detailed information on the dynamics of solidliquid interface and temperature fields [1-4].

Despite the action of natural convection, when free liquid surface is present in the melting process, Marangoni flow induced by the surface tension gradient along the interface could also be considered to be significant. Lin et al. [5] numerically investigated the flow and temperature fields during ice melting, considering the combined effects of heat conduction, natural convection, and the Marangoni flow. These authors noted that, although heat conduction has played a predominant role, the Marangoni flow could also affect the melting process, especially on the shape of the solidliquid interface. Natural convection has become insignificant in their sample calculations.

All numerical simulations should begin with an initial molten pool. Previous studies arbitrarily 


\begin{tabular}{|c|c|c|c|}
\hline \multicolumn{4}{|c|}{ Nomenclature } \\
\hline$A$ & area $\left(\mathrm{m}^{2}\right)$ & $T_{S}$ & steady-state temperature distribution $(\mathrm{K})$ \\
\hline$B$ & parameter defined in Eq. $(21 \mathrm{a}, \mathrm{b})$ & $\Delta T$ & maximum temperature difference $(\mathrm{K})$ \\
\hline$B_{C}$ & critical $B$ value & $t$ & time $(\mathrm{s})$ \\
\hline Bo & bond number & $u$ & component of velocity along $x$-axis $\left(\mathrm{m} \mathrm{s}^{-1}\right)$ \\
\hline$C$ & constant defined in Eq. (13b) & $\vec{V}$ & velocity of liquid $\left(\mathrm{m} \mathrm{s}^{-1}\right)$ \\
\hline$C_{p}$ & specific heat $\left(\mathrm{J} \mathrm{kg}^{-1} \mathrm{~K}^{-1}\right)$ & $v$ & component of velocity along $y$-axis $\left(\mathrm{m} \mathrm{s}^{-1}\right)$ \\
\hline$F$ & $\begin{array}{l}\text { perturbed function defined in Eqs. (18a) } \\
\text { and (18b) }\end{array}$ & $X_{\max }$ & $\begin{array}{l}\operatorname{maximum} \text { interfacial position on } x \text {-axis } \\
(\mathrm{m})\end{array}$ \\
\hline$f$ & perturbed function defined in Eq. (18a) & $x_{1}$ & computational domain along the $x$-axis \\
\hline$g$ & perturbed function defined in Eq. (18b) & & $(\mathrm{m})$ \\
\hline$g$ & gravitational acceleration $\left(\mathrm{m} \mathrm{s}^{-2}\right)$ & $Y_{\max }$ & maximum interfacial position on the $y$-axis \\
\hline$k$ & thermal conductivity $\left(\mathrm{W} \mathrm{m}{ }^{-1} \mathrm{~K}^{-1}\right)$ & & $(\mathrm{m})$ \\
\hline$L$ & latent heat $\left(\mathrm{J} \mathrm{kg}^{-3}\right)$ & $y_{1}$ & computational domain along the $y$-axis (m) \\
\hline$m$ & surface heat flux gradient along the liquid & \multirow{2}{*}{\multicolumn{2}{|c|}{ Greek symbols }} \\
\hline & surface $\left(\mathrm{W} \mathrm{m}^{-3}\right)$ & & \\
\hline$M a$ & Marangoni number & $\alpha$ & thermal diffusivity $\left(\mathrm{m}^{2} \mathrm{~s}^{-1}\right)$ \\
\hline$p$ & pressure $(\mathrm{Pa})$ & $\beta$ & volume expansion coefficient $\left(\mathrm{K}^{-1}\right)$ \\
\hline $\mathrm{Pr}$ & Prandtl number & $\Gamma$ & $\alpha t / \delta^{2}$ \\
\hline$Q$ & heat flow rate $(\mathrm{W})$ & $\gamma$ & kinematic viscosity $\left(\mathrm{m}^{2} \mathrm{~s}^{-1}\right)$ \\
\hline$Q_{f}$ & heat transfer intensity defined by Eqs. (13a) & $\lambda$ & eigenvalue \\
\hline & and $(13 b)$ & $\delta$ & thickness of the molten pool (m) \\
\hline$q_{0}^{\prime \prime}$ & imposed surface heat flux $\left(\mathrm{W} \mathrm{m}^{-2}\right)$ & $\delta_{C}$ & critical thickness of the molten pool (m) \\
\hline & heat flux along the interface $\left(\mathrm{W} \mathrm{m}^{-2}\right)$ & $\theta$ & excess temperature $(=T-T f)(\mathrm{K})$ \\
\hline$R a$ & Rayleigh number & $\omega$ & variable in separation of variables \\
\hline$r_{0}$ & radius of heat source $(\mathrm{m})$ & $\mu$ & viscosity (Pa s) \\
\hline$S$ & interfacial displacement $(\mathrm{m})$ & $\tau$ & function defined in Eq. (8a) \\
\hline$T$ & temperature $(\mathrm{K})$ & $\rho$ & density $\left(\mathrm{kg} \mathrm{m}^{-3}\right)$ \\
\hline & perturbed temperature $(\mathrm{K})$ & $\xi$ & function defined in Eq. (8b) \\
\hline & melting temperature $(\mathrm{K})$ & $\zeta$ & $x / \delta$ \\
\hline$T_{\text {mean }}$ & bulk mean temperature $(\mathrm{K})$ & $\eta$ & $y / \delta$ \\
\hline$T_{0 B}$ & steady-state liquid temperature at the bot- & $\sigma$ & surface tension $\left(\mathrm{N} \mathrm{m}^{-1}\right)$ \\
\hline & tom $(\mathrm{K})$ & & reference surface tension $\left(\mathrm{N} \mathrm{m}^{-1}\right)$ \\
\hline$T_{0 S}$ & $\begin{array}{l}\text { steady-state liquid temperature at the sur- } \\
\text { face }(\mathrm{K})\end{array}$ & $\Xi$ & function defined in Eq. $(21 \mathrm{a}, \mathrm{b})$ \\
\hline
\end{tabular}

assume the shape of an initial pool [6-8], or adopt conduction heat transfer mechanism to construct an initial molten pool $[9,10]$. Lin et al. [5] examined the effects of adopting different initial molten pools to numerical solutions, and noted that different initial molten pool would yield a different calculated melting process. Restated, an arbitrarily assumed initial molten pool could lead to erroneous numerical results during solid melting.

A realistic formulation to the initial molten pool is desired, which is the main theme of this study. In the subsequent sections we would first list the governing equations for the melting process and the solution logic. At the very beginning the melting process should be controlled by heat conduction alone. Fluid motion could occur after a molten pool of finite thickness was formed. We then analyzed the linear stability charac- teristics of a molten pool formed by heat conduction melting that is subject to Marangoni flow induced by the perturbations of surface liquid temperature. The critical time for the melting from purely conductive process to incorporate fluid motion was evaluated. The initial molten pool could hence be properly formulated for numerical solution. Finally we examined the validity of the proposed initial molten pool to numerical solution.

\section{Equations and solution}

\subsection{Governing equations}

Fig. 1 schematically depicts the melting process. The governing equations of the transport process in the 
molten pool are as follows:

$\nabla \cdot \vec{V}=0$

$\frac{\partial \vec{V}}{\partial t}+\vec{V} \cdot \nabla \vec{V}=-\frac{1}{\rho} \nabla p+\gamma \nabla^{2} \vec{V}$

$\frac{\partial T}{\partial t}+\vec{V} \cdot \nabla T=\alpha \nabla^{2} T$

Here we considered the two-dimensional case of Eqs. (1)-(3) where $\vec{V}=(u, v)$ and $u$ and $v$ are the velocity components along the $x$ - and $y$-axis, respectively.

A heat flux of $q_{0}^{\prime \prime}$ is imposed onto a circular upper surface of radius $r_{0}$. Other surfaces assumed to be are insulated. Furthermore, Marangoni flow is induced by the surface tension gradient along the free liquid surface. The boundary conditions on the free liquid surface are as follows:

$q_{0}^{\prime \prime}=-k \frac{\partial T}{\partial y}, \quad$ at $y=0, \quad 0<x<r_{0}$

$q_{0}^{\prime \prime}=0, \quad$ at $y=0, \quad r_{0}<x<r_{1}$

$\tau=-\mu \frac{\partial u}{\partial y}=\left(\frac{\mathrm{d} \sigma}{\mathrm{d} T}\right)\left(\frac{\partial T}{\partial x}\right), \quad$ at $y=0$,

$0<x<x_{1}$

The boundary conditions at the solid-liquid interface are as follows:

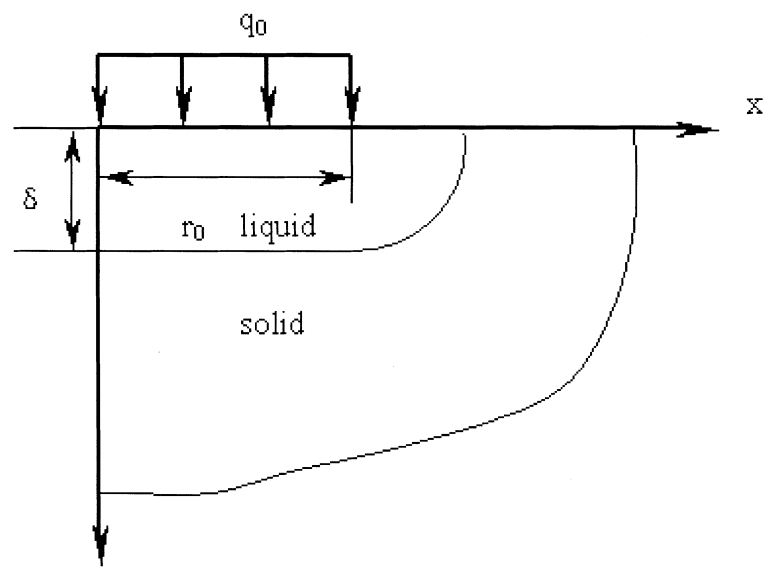

y $\mathrm{d} Q=\rho A L \frac{\partial S}{\partial t}, \quad$ and $\quad T=T_{f}$

The symmetrical conditions include:

$\frac{\partial v}{\partial x}=\frac{\partial v}{\partial x}=u=0, \quad$ at $x=0, \quad 0<y<y_{1}$.

$(6 a-c)$

Eqs. (1)-(3), together with boundary conditions, Eqs. (4a), (4b), (4c), (5a,b) and $(6 a-c)$, would be completed if an initial condition is applied. The initial molten pool was discussed in the next section.

\subsection{Heat-conduction controlled process}

Let $\vec{V}=0$, the melting process becomes heat-conduction controlled. Further assume that the molten pool thickness is much less than its radius, then the transport process could be taken as one-dimensional. Using pseudo-steady state approximation, an analytical solution relating the location of interface and the melting time are as follows [11]:

$\tau=\frac{\xi}{6}\left[\xi+5+(1+4 \xi)^{1 / 2}\right]$,

where

$\xi=\frac{q(t) \delta(t)}{\alpha \rho L}$,

and

$\tau=\frac{q(t)}{\rho^{2} \alpha L^{2}} \int_{0}^{1} q(t) \mathrm{d} t$.

Fig. 2 illustrates the $\xi$ versus $\tau$ curve, which provides a unique correlation between the pool thickness and melting time. Very clearly the heat conduction could be regarded as the predominant heat transfer mechanism at the beginning stage.

\subsection{Numerical solution}

Complete solution of Eqs. (1)-(3), (4a), (4b), (4c), $(5 \mathrm{a}, \mathrm{b})$ and $(6 \mathrm{a}-\mathrm{c})$ could be solved numerically if the initial condition was available. The numerical method proposed by Lin et al. [5] has been adopted herein. That is, the pseudo-steady state velocity and temperature fields are calculated at small time increments with the assistance of the CFD package PHOENICS. The evolution of solid-liquid interface was adjusted at each time increment. The residuals for the continuity equation and momentum equations are less than $10^{-5}$ and the residuals for the energy equation were less than $10^{-2}$. If the numerical outcome deviates from that of Eq. (7), heat mechanisms other than heat conduction should incorporate and play an essential role in the melting process. 


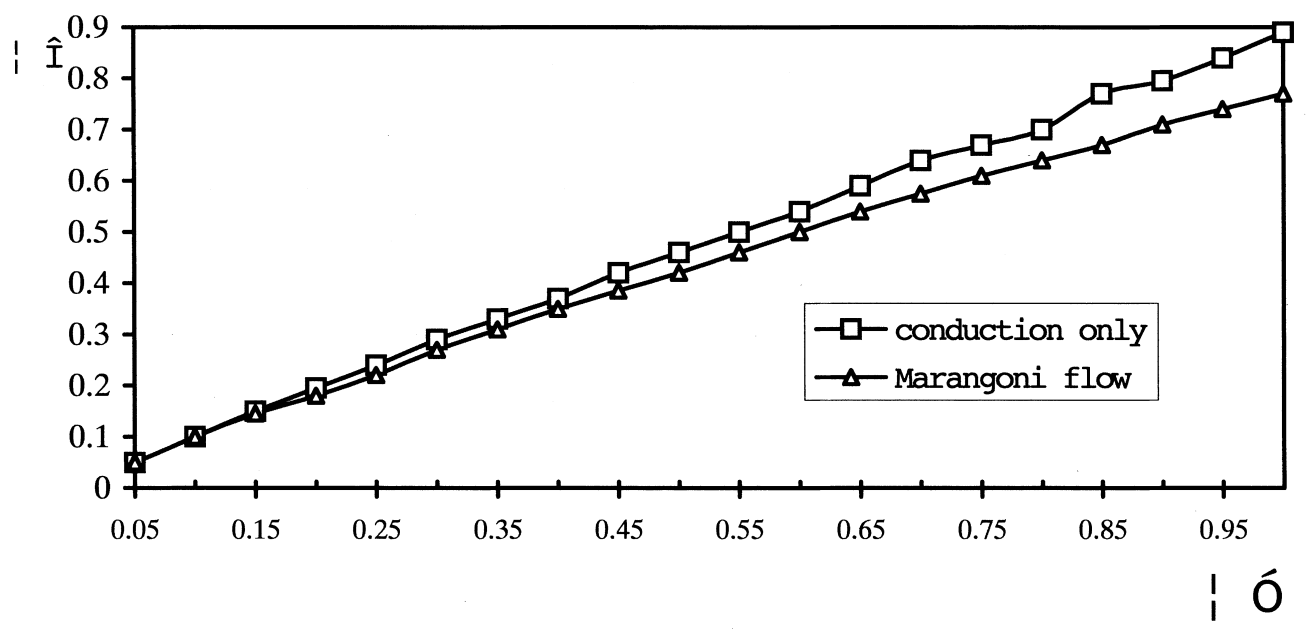

Fig. 2. $\xi$ vs. $\tau$ curve.

\section{Critical conditions for conduction-controlled melting}

\subsection{Evaluation of the critical time}

The Marangoni number $(M a)$, denoting the ratio between heat flux induced by Marangoni flow and the conduction heat flux, could be stated as follows:

$M a=\left|\frac{\mathrm{d} \sigma}{\mathrm{d} T}\right| \frac{L r_{0}}{C_{p} \alpha \mu}=\left|\frac{\mathrm{d} \sigma}{\mathrm{d} T}\right| \frac{\delta \Delta T}{\alpha \mu}$,

where $\Delta T$ is the maximum temperature difference occurring in the system. Meanwhile, the Rayleigh number $(R a)$ controls the intensity of natural convection, which is defined as follows:

$R a=\frac{g \beta \Delta T \delta^{3}}{\gamma \alpha}$

The ratio $R a / M a$ defines the Bond number $(B o)$ :

$B o=\left|\frac{g \beta \rho \delta^{2}}{(\mathrm{~d} \sigma / \mathrm{d} T)}\right|$.

Notably, if $B o \ll 1$, the role of Marangoni flow would be much more significant than that of natural convection.

At the very beginning of melting the heat conduction should be the only dominant mechanism. In the subsequent stage other mechanisms like Marangoni flow, might become significant. It becomes essential to estimate the critical time for the occurrence of transition from purely conductive process to a combinedmechanism process.

The procedures are as follows. First, the thickness of a molten pool $(\delta)$ heated from top surface at heat flux of $q_{0}^{\prime \prime}$ by purely conductive process could be estimated according to Eq. (7) in a very short period of time. The radius of molten pool is $r_{0}$, satisfying $\delta \ll r_{0}$. As a result, the heating process could be approximately semi-infinite. Then the boundary condition considering Marangoni flow (Eq. (4c)) was imposed upon the free liquid surface to evaluate the new radius of the molten pool, $r_{0}^{\prime}$. By the equality $q r_{0}=q^{\prime} r_{0}^{\prime}$, we could evaluate the new heat flux at $q^{\prime}$, whence evaluating the surface area of the molten pool $\Sigma \mathrm{A}$ and the new pool thickness $\delta^{\prime}$. Table 1 lists the parameters adopted herein in sample calculations. The working material is water. Since the estimated $\delta$ values are about $7 \%$ of the diameter of the heating area $\left(2 r_{0}=5 \mathrm{~mm}\right)$, the semi-infinite domain assumption adopted herein has become a reasonable approximation to solution.

Fig. 2 also illustrates the $\xi$ versus $\tau$ curve for the case with combined effects of heat conduction and Marangoni flow. Notably this curve deviates from that of pure conduction mechanism at $\tau=0.22$, corresponding to a melting time of $5.4 \mathrm{~s}$ and a melt thickness of $0.37 \mathrm{~mm}$. Restated, at a melting time exceeding this

Table 1

Parameters adopted in sample calculations

\begin{tabular}{ll}
\hline$q_{0}^{\prime \prime}$ & $1.0 \mathrm{E} 6 \mathrm{~W} \mathrm{~m}^{-2}$ \\
$r_{0}$ & $0.005 \mathrm{~m}$ \\
$C_{p}$ & $4180 \mathrm{~J} \mathrm{~kg}^{-1} \mathrm{~K}^{-1}$ \\
$\rho$ & $999.9 \mathrm{~kg} \mathrm{~m}^{-3}$ \\
$P r$ & 7.01 \\
$k$ & $0.598 \mathrm{~W} \mathrm{~m}^{-1} \mathrm{~K}^{-1}$ \\
$L$ & $3.35 \mathrm{E} 5 \mathrm{~J} \mathrm{~kg}^{-1}$ \\
$\beta$ & $1.74 \mathrm{E} 4 \mathrm{~K}^{-1}$ \\
$T_{f}$ & $273.15 \mathrm{~K}^{-1} \mathrm{~s}^{-1}$ \\
$\mu$ & $1.0 \mathrm{E} 6 \mathrm{~kg} \mathrm{~m}^{-1}$ \\
$\sigma$ & $0.147 \mathrm{mN} \mathrm{m}^{-1}$ \\
\hline
\end{tabular}


critical time the process could not be regarded as controlled solely by heat conduction mechanism. This critical time had been employed in the following sections to evaluate the effects of arbitrarily assumed initial molten pool on the simulations of melting process.

\subsection{Effects of shape of initial pool}

As the previous section reveals, the Marangoni flow would become effective in the melting process as $t$ exceeds $5.4 \mathrm{~s}$. We constructed three initial molten pools (pools I-III) formed by pure heat conduction effected at $t=2,5$, and $10 \mathrm{~s}$, respectively. According to the critical time estimated previously, the conduction-controlled assumption is valid for pool I, but flawed for pool III. For pool II, it is just at the transition stage.

Fig. 3a schematically depicts the molten pool. The semi-infinite assumption could be adopted to estimate the pool thickness. The corresponding $\delta / r_{0}$ ratios for pool I to III are $0.0288,0.0688$, and 0.130 , respectively. The temperature distribution in the molten pool by purely heat conduction mechanism could be expressed as follows:

$$
\begin{aligned}
\frac{\theta(y, \tau)}{L / C_{p}}= & \frac{1}{2}\left[1-(1+4 \xi)^{1 / 2}\right]\left(\frac{y}{\delta}-1\right)+\frac{1}{8}[1-(1 \\
& \left.+4 \xi)^{1 / 2}\right]^{2}\left(\frac{y}{\delta}-1\right)^{2}
\end{aligned}
$$

Meanwhile, the velocity in the pool is defined as zero (conduction-controlled). The molten pool of thickness $\delta$, together with the temperature field predicted by Eq. (12), represents the initial molten pool for numerical investigation.

In Fig. 3a only the solid under the circular area of radius $r_{0}$ is affected by the surface heat flux. However, in reality, the imposed surface heat flux, $q_{0}^{\prime \prime}$, should not merely affect the solid within $r<r_{0}$, but also influence the temperature distributions of solid phase adjacent to the edge as well the side interfacial shape of the molten pool. We model the edge as a circular region for more realistic description as depicted in Fig. 3b.

Notably, by setting $\vec{V}=0$, Eqs. (1)-(3) represent a purely conductive process. Moreover, $\nabla p=0$ in Eq.
(2) or $\mathrm{d} \sigma / \mathrm{d} T=0$ in Eq. (4c) could switch off the natural convection or the Marangoni flow, respectively.

\section{Linear stability analysis of the molten pool}

\subsection{Linear temperature profile}

To explore the linear stability characteristics of an initial molten pool subject to the Marangoni flow induced by surface liquid temperature fluctuations, consider an infinitely larger molten liquid of thickness $\delta$ with a uniform heat flux of $q_{0}^{\prime \prime}$ imposing onto the upper surface. The governing equations of the transport process in the molten pool (Newtonian fluid) neglecting the natural convection effect are listed in Eqs. (1)-(3). The one-dimensional, steady-state, noflow solutions of Eqs. (1)-(3), (4a), (4b) and (4c) are as follows:

$u=v=0$,

$T_{S}=T_{0 B}-C y$

where $C<0$, and $q_{0}{ }^{\prime \prime}=k C$. Eqs. (13a) and (13b) represent a pure heat-conduction controlled process at which $\partial \sigma / \partial x=0$.

Small temperature perturbation $T^{\prime}$ was imposed to the steady-state solution Eq. (13b). Specify the following constraints to the small perturbation $T^{\prime}: \partial T^{\prime} / \partial x \neq 0$ on the free liquid surface $(y=\delta)$, and $\partial T^{\prime} / \partial x=0$ inside the molten pool $(0<y<\delta)$. The non-zero velocity components, $u$ and $v$ are the perturbed velocities.

The linearized equations derived from Eqs. (1)-(3) include:

$\left(\frac{\partial}{\partial t}-\gamma \nabla^{2}\right) v=0$

$\left(\frac{\partial}{\partial t}-\alpha \nabla^{2}\right) T^{\prime}=C v$

Assume that the surface tension changes linearly with temperature, that is,

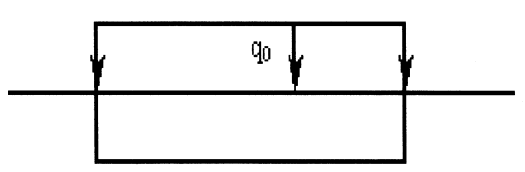

(a)

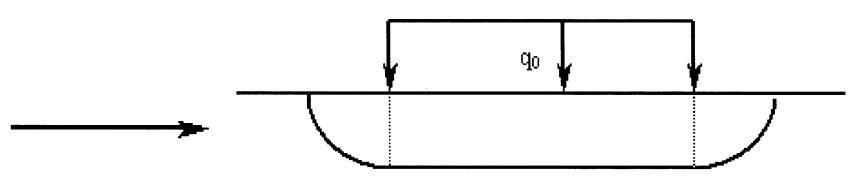

(b)

Fig. 3. (a) Initial molten pool; (b) modified initial molten pool. 
$\sigma=\sigma_{0}+\frac{\mathrm{d} \sigma}{\mathrm{d} T} T^{\prime}$

The surface heat flux would also change as follows:

$q=q_{0}+m T^{\prime}$

where $m=(\partial q / \partial T)_{T=T_{0 S}}$.

The associated boundary conditions are as follows:

$\frac{\partial T^{\prime}}{\partial y}=0, \quad k \frac{\partial T^{\prime}}{\partial y}=m T^{\prime} \quad$ at $y=0$

$-\mu \frac{\partial^{2} u}{\partial y^{2}}=\frac{\mathrm{d} \sigma}{\mathrm{d} T} \frac{\partial^{2} T^{\prime}}{\partial x^{2}}, \quad v=0 \quad$ at $y=\delta$

Introducing the dimensionless groups $(\zeta, \eta)=(x / \delta, y /$ $\delta$ ) and $\Gamma=\alpha t / \delta^{2}$ into the perturbed equations, and letting

$v=\frac{\alpha}{\delta} F(\zeta) f(\eta) \mathrm{e}^{\lambda \Gamma}$,

and

$T^{\prime}=-C F(\zeta) g(\eta) \mathrm{e}^{\lambda \Gamma}$

lead to the following equations:

$\left\lfloor\lambda+\operatorname{Pr}\left(D^{2}-\omega^{2}\right) \perp D^{2}-\omega^{2}\right\rfloor f=0$,

$\left\lfloor\lambda+\operatorname{Pr}\left(D^{2}-\omega^{2}\right)\right\rfloor g=-f$,

where $D=\mathrm{d} / \mathrm{d} y$ and $\omega$ represents the dimensionless variable appearing in separation of variables. The corresponding boundary equations are as follows:

$$
\begin{aligned}
& f(0)=f^{\prime \prime}(0)=0 ; \quad g(0)=0 \\
& f(1)=0, f^{\prime \prime}(1)=\omega^{2} B g(1), \quad \text { and } \\
& g^{\prime}(1)=\Xi g(1) .
\end{aligned}
$$

The parameters in Eq. $(20 \mathrm{a}-\mathrm{e})$ are defined as follows:

$B=\frac{(\mathrm{d} \sigma / \mathrm{d} T) \beta \delta^{2}}{\alpha \mu}$ and $\Xi=\frac{m \delta}{k}$.

The neutral stability condition for Eqs. (19a) and (19b) could be obtained by settling $\lambda=0$. Restated,

$\operatorname{Pr}\left(D^{2}-\omega^{2}\right)\left(D^{2}-\omega^{2}\right) f=0$,

$\operatorname{Pr}\left(D^{2}-\omega^{2}\right) g+f=0$.

Pearson [12] provided the solutions for Eqs. (22a) and (22b) together with the boundary conditions Eq. (20a-e). The following for $f$ and $g$ are as follows:

$$
\begin{aligned}
f= & \omega\left(\sinh \omega \eta+\frac{\omega \cosh \omega-\sinh \omega}{\sinh \omega} \eta \sinh \omega \eta\right. \\
& -\omega \eta \cosh \omega \eta)
\end{aligned}
$$

$$
\begin{aligned}
g= & \omega\left(\frac{3}{4 \omega} \eta \cosh \omega \eta\right. \\
& +\frac{\omega \cosh \omega-\sinh \omega}{4 \omega \sinh \omega} \eta^{2} \sinh \omega \eta \\
& -\frac{1}{4} \eta^{2} \sinh \omega \eta \\
& -\frac{\omega \cosh \omega-\sinh \omega}{4 \omega^{2} \sinh \omega} \eta \sinh \omega \eta \\
& \left.-\frac{3}{4 \omega^{2}} \sinh \omega \eta\right)
\end{aligned}
$$

Substitute the solutions of $f$ and $g$ into Eq. (20a-e) yields the expression of $B$ as follows:

$B=$

$8 \omega(\omega \sinh \omega+h \cosh \omega)(\omega-\sinh \omega \cosh \omega)$

$\left(\omega^{3} \sinh \omega-\omega^{2} \cosh \omega+2 \omega \sinh \omega-\sinh ^{2} \omega \cosh \omega\right)$.

The critical value of $B$ could hence be evaluated as $B_{c}=80$. The critical liquid film thickness could be evaluated with the assistance of definition of $B$ in Eq. (21a,b) as follows:

$\delta_{C}=\sqrt{\frac{80 \alpha \mu}{|(\mathrm{d} \sigma / \mathrm{d} T)(\partial T / \partial y)|}}$.

Taking $\partial T / \partial y \approx \Delta T / \delta_{C}$, Eq. (25) could be rearranged into:

$\delta_{C}=\frac{80 \alpha \mu}{|(\mathrm{d} \sigma / \mathrm{d} T)| \Delta T}$.

At a liquid film thickness exceeding $\delta_{C}$, the molten pool would become unstable when subjected to surface temperature perturbation, $T^{\prime}$. Substituting the related parameters into Eq. (26) could estimate the critical film thickness. For the present case, the critical thickness is estimated to be $0.013-0.13 \mathrm{~mm}$ at $\Delta T=1-10$ $\mathrm{K}$. The numerical solutions provided in the preceding sections suggests a critical thickness of approximately $0.37 \mathrm{~mm}$. The present linear stability analysis gives a smaller $\delta_{C}$. Such an observation might come from the inconsistent, linear temperature distribtution proposed in Eq. (13b). The latter point will be discussed in the next section. 


\subsection{Parabolic temperature profile}

We herein replaced Eq. (13a) by the parabolic temperature profile listed in Eq. (12) to re-derive the stab- ility criterion based on this profile. Since the slopes of liquid temperature along vertical distance differ over the entire liquid film, different critical film thickness could be evaluated. If the slope is taken at free surface
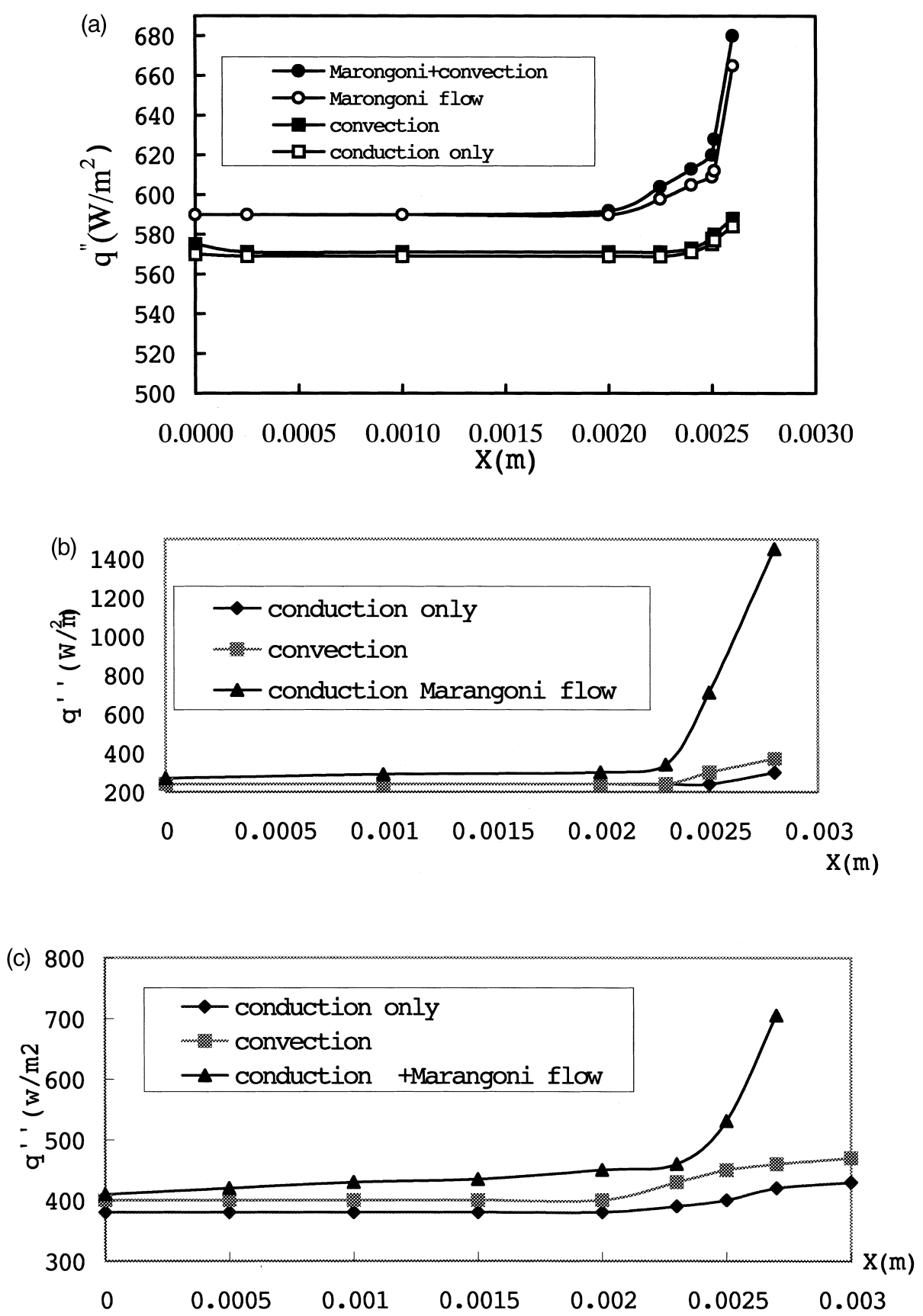

Fig. 4. (a) Heat fluxes along the free liquid surface for pool I; (b) heat fluxes along the free liquid surface for pool II; (c) heat fluxes along the free liquid surface for pool III. 


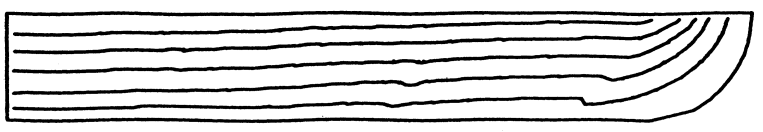

(b)

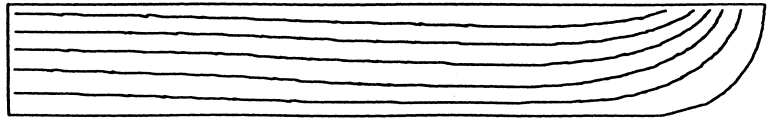

(a)

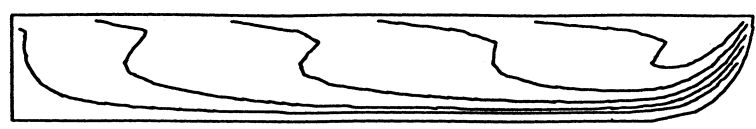

(c)

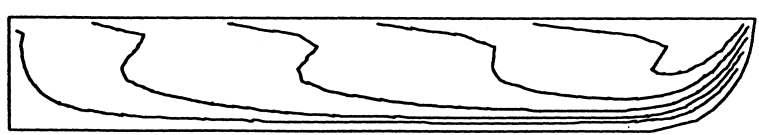

(d)

Fig. 5. (a) Isothermal lines for pool II considering heat conduction alone; (b) isothermal lines for pool II considering heat conduction and convection; (c) isothermal lines for pool II considering three kinds of heat transfer mechanisms; (d) isothermal lines for pool II considering three kinds of heat transfer mechanisms and Marangoni flow.

in the calculation, the results is as follows:

$\delta_{C}=\sqrt{\frac{80 \alpha \mu}{|(\mathrm{d} \sigma / \mathrm{d} T)|\left(q / C_{p} \alpha \rho\right)}}$.

If, on the other hand, taking the slope at the liquidsolid interface in calculation, the result is:

$\delta_{C}=\frac{80 \alpha \mu C_{p}}{|(\mathrm{~d} \sigma / 2 \mathrm{~d} T)|\left|1-(1+4 \xi)^{1 / 2}\right| L}$,

where $\xi$ is a function of $\delta_{C}$.

Substituting related data in Eqs. (27a) and (27b), yields the critical film thickness of 0.053 and 0.078 $\mathrm{mm}$, respectively. Consequently, the assumed temperature distribution does not correspond with the deviation between linear stability analysis and the numerical solutions. The inherent limitations of linearized analysis should correlate with the deviations.

\section{Effects of initial molten pool}

\subsection{Initial dynamics of melting}

In this section the term 'initial dynamics' denotes the melting process just after several integration time steps from the initial pool. Restated, the initial melting times for pools I-III are different, namely slightly exceeding 2,5 , and $10 \mathrm{~s}$, respectively.

Fig. $4 \mathrm{a}-\mathrm{c}$ compare the heat fluxes along the free liquid surface considering conduction only, conduction + convection, conduction + Marangoni flow, and the combined effects of the three mechanisms. There are three points to be noted. First, heat conduction dominates the processes during the initial phase of melting. Also, the effect of Marangoni flow is much more significant than that of natural convection, which correlates with the results of Lin et al. [5]. Second, comparing Fig. 4a and $\mathrm{b}$, since the molten pool is rather thin, the action of Marangoni flow mainly enhances the heat flow close to the rim regime rather than that at the central regime. A longer conduction time reduces the heat flux owing to the thicker liquid pool thickness. Third, the trends depicted in Fig. 4c differ from those in Fig. $4 a$ and $b$, revealing that to adopt pool III as the initial condition for simulation may be erroneous.

Fig. 5a-d illustrate the isothermal lines for pool II just after the start of melting (somewhat exceeding 5 s), considering various combinations of heat transfer mechanisms. The isothermal lines owing to heat conduction alone are all closely parallel to each other (Fig. 5a). However, the incorporation of Marangoni flow markedly distorts the isothermal lines (Fig. 5c and d). The isothermal lines at the rim regime become more crowded, indicating a stronger heat flux at that regime. Moreover, the high-temperature fluid would

(a)

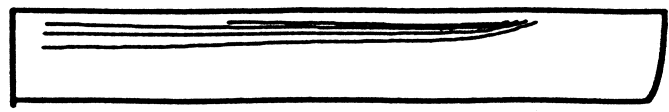

(b)

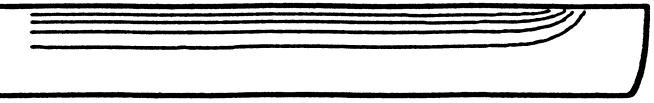

Fig. 6. (a) Isothermal lines for pool I considering heat conduction only; (b) isothermal lines for pool I considering heat conduction and Marangoni flow. 


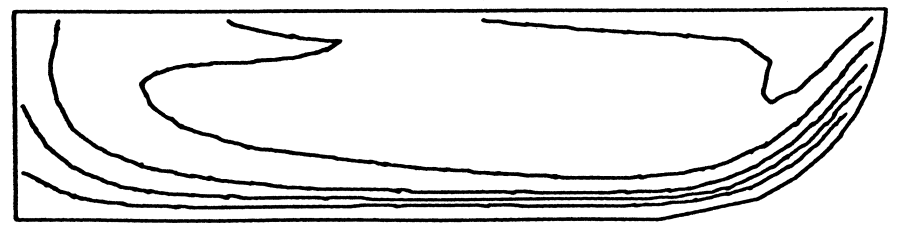

Fig. 7. Isothermal lines for pool III considering Marangoni flow.

'penetrate' into the deeper layers of the fluid owing to the Marangoni flow.

Fig. $6 \mathrm{a}$ and $\mathrm{b}$ depict the isothermal lines for pool I just after the start of melting, that is, somewhat exceeding 2 s. Since such a melting time is less than the critical time $(5.4 \mathrm{~s})$ for the process, Marangoni flow should not have come into play. As clearly demonstrated in Fig. 6a and b, we note that Marangoni flow really does not affect the isothermal lines, thereby having a negligible role in melting.

Fig. 7 demonstrates the isothermal lines for pool III just after the start of melting, that is, somewhat exceeding $10 \mathrm{~s}$. Since the time adopted largely exceeds the critical time, the effects of Marangoni flow has affected the temperature distributions over the whole molten pool. The temperature in the pool has become more uniform owing to the mixing effect induced by the Marangoni flow.

Fig. $8 \mathrm{a}-\mathrm{c}$ depict the velocity fields for pools I-III just after the start of their corresponding melting time. For pool I (Fig. 8a), the fluid flow field has not been well developed. Weak flow exists close to the rim regime. For pool II (Fig. 8b), the velocity field has been fully developed, with its maximum velocity approximately three times to that for pool I. The velocity field becomes much stronger for pool III (Fig. 8c) than those for pools I and II. Apparently, using pool III as the initial condition for numerical simulation would overestimate the role of Marangoni flow.

Fig. $9 \mathrm{a}-\mathrm{c}$ demonstrate the liquid temperature along the liquid surface for pools I-III after the start of melting. For pool I, the presence of Marangoni flow lowers the surface temperature when compared with the case considering only heat conduction. Nevertheless, the basic characteristic of temperature distribution is not affected by the Marangoni flow. For pools II and III, nevertheless, the surface temperature distributions exhibit different characteristics when Marangoni flow comes into play. The temperature at the central regime would increase with radius, in contradiction to the pure conduction case. The low temperature of surface liquid is attributed to the mixing of liquid close to the central regime with the coming bottom liquid from the rim region. When the liquid flows outward along the upper surface, its temperature would increase owing to the surface heat flux.

In all the cases investigated above, during the initial stage after the simulation, pools I and II gave identical results. This correlation agrees with the predicted performance in previous sections that the heat conduction controlled assumption is valid for initial pools I and II. Pool III gives erroneous numerical results since the assumed conduction-controlled period is too long. Moreover, the maximum velocity induced by natural

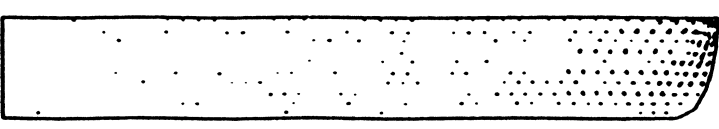

(a)

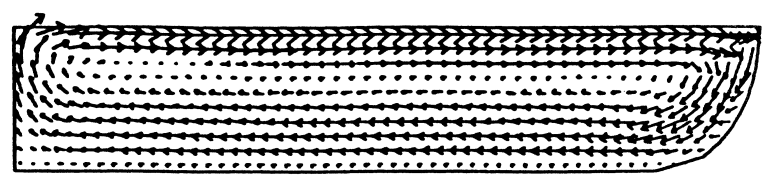

(b)

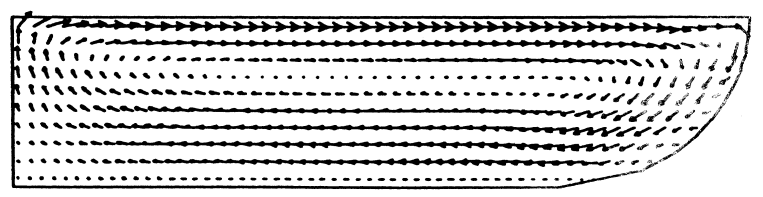

(c)

Fig. 8. (a) Velocity fields for pool I; (b) velocity fields for pool II; (c) velocity fields for pool III. 


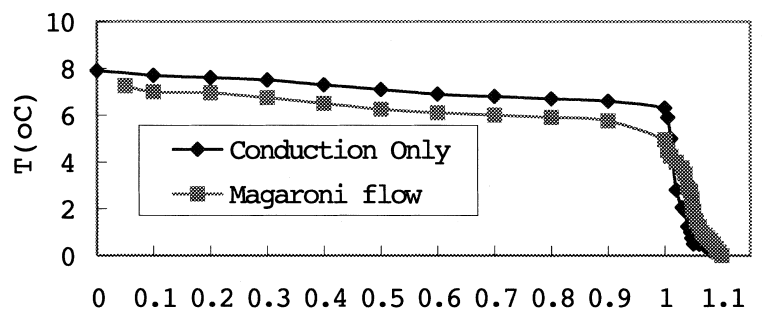

(a)

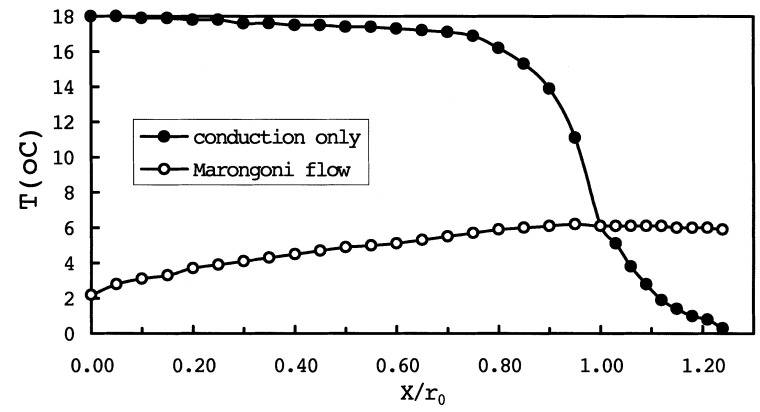

(b)

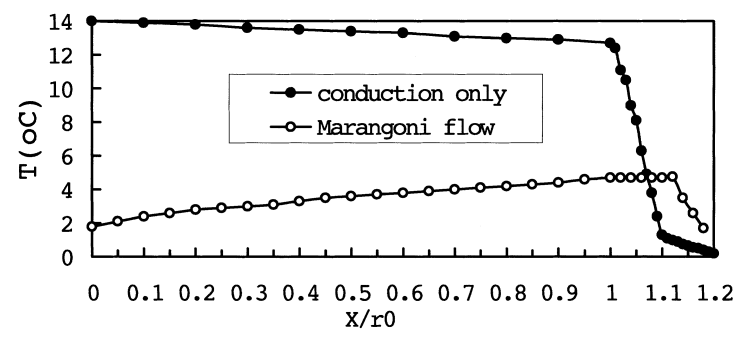

(c)

Fig. 9. (a) Liquid temperature along free surface for pool I; (b) liquid temperature along free surface for pool II; (c) liquid temperature along free surface for pool III.

convection is two to three orders of magnitude less than that by Marangoni flow. Marangoni flow affects mainly the fluid flow and temperature distributions near the rim region.

\subsection{Long-term dynamics}

For demonstrating the long-term effects, Fig. 10a and $b$ illustrate the shapes of solid-liquid interface and the heat fluxes at the interface at $t=30 \mathrm{~s}$ for the three initial pools. Notably, the results for pool I and II still closely correlate with each other, indicating an insignificant role of the initial pool. Pool III assumes a too-

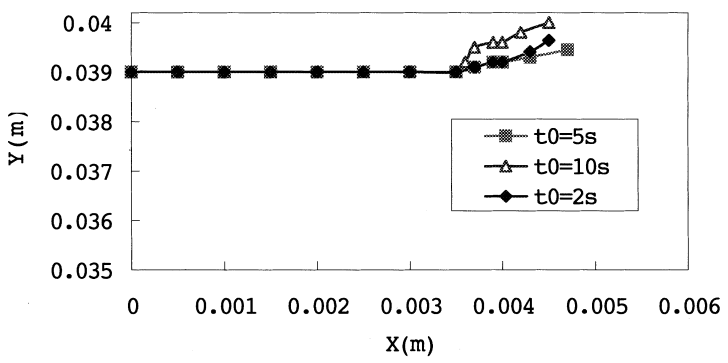

(a) long melting time for a pure conduction-controlled process. In doing so, at $30 \mathrm{~s}$, the molten pool would exhibit a smaller radius and a different heat flux distribution at the liquid surface.

Fig. 11a and $\mathrm{b}$ illustrate the temperature and velocity along the free liquid surface for pool II just after the start of melting and at $t=30 \mathrm{~s}$, respectively. At short time of melting, where $r=1.2 r_{0}$, the surface temperature increases with increasing radius, reaching a maximum at the edge of heated area, then decreases when moving further outward. The corresponding surface velocity, on the other hand, increases in the central region, reaching a maximum value at the edge

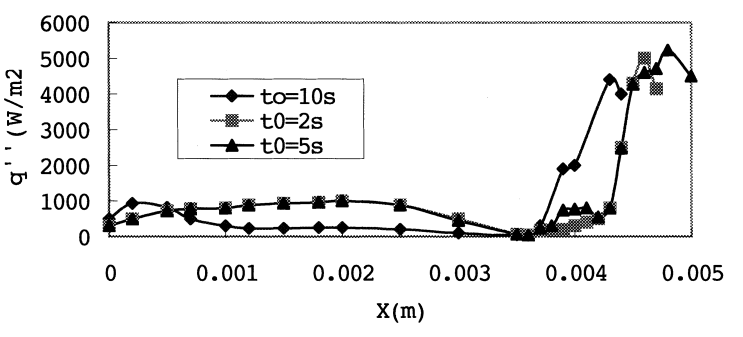

(b)

Fig. 10. (a) Solid-liquid interfacial shape at $t=30 \mathrm{~s}$ for the three initial pools; (b) heat fluxes at the interface at $t=30 \mathrm{~s}$ for the three initial pools. 


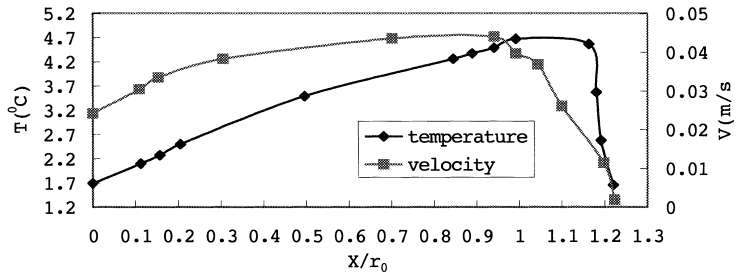

(a)

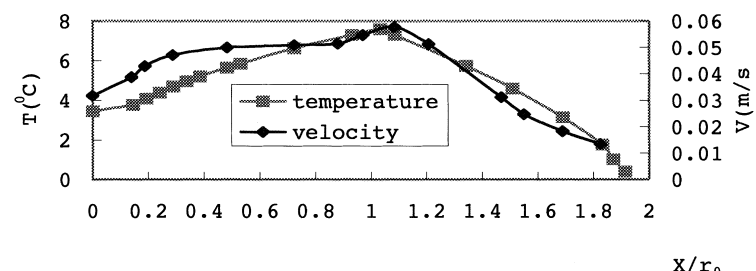

(b)

Fig. 11. (a) Temperature and velocity along the free liquid surface for pool II just after the start of melting; (b) temperature and velocity along the free liquid surface for pool II at $t=30 \mathrm{~s}$.

region, then droping to zero to the solid-liquid interface.

After $30 \mathrm{~s}$, the radius of molten pool reaches $1.8 r_{0}$. Both temperature and velocity distributions closely correspond with each other, and reach their maximum values at the edge of the heated area.

Fig. 12 depicts the boundaries of molten pool along $x$ - and $y$-axis on the basis of initial molten pool II. Notably, during the initial stage of melting the molten pool mainly develops along the $x$-axis, with its maximum thickness $\left(Y_{\max }\right)$ almost invariant. Such an occurrence is attributed to the induced flow by Marangoni flow that enhances the heat flux at the rim region. The melting would hence be effective along the radial direction. In the subsequent stage, heat transfer enhancement regime moves to the symmetrical axis. The thickness of molten pool hence largely increases after $40 \mathrm{~s}$. The increasing rate of the corresponding radius of molten pool $\left(X_{\max }\right)$ becomes lesser.

To clearly demonstrating the changes in heat flux at the solid-liquid interface and liquid temperature along free liquid surface, Fig. 13a and b depict their distributions at $t=3,40$, and $70 \mathrm{~s}$, respectively, for initial pool I. Marked differences are noted for these trends. Fig. 14a-c illustrates the corresponding velocity fields of fluid. Comparing Figs. 13 and 14 clearly reveal the effects of induced fluid flow field on the detailed shape and the corresponding heat flux/surface temperature distributions.

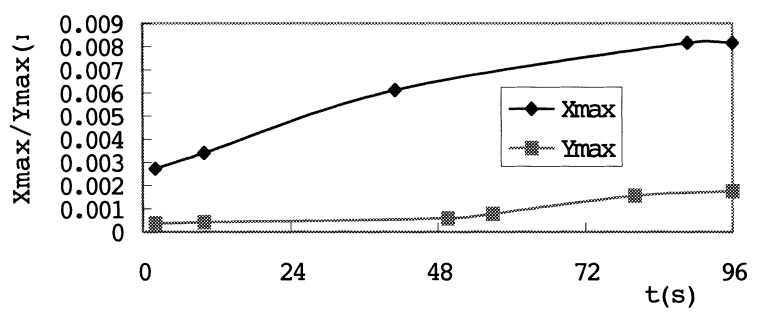

Fig. 12. Boundaries of molten pool along $x$ - and $y$-axis on the basis of initial molten pool II.

\subsection{Effects of heat transfer intensity}

In Eq. (8a) the dimensionless group, $\xi$, is defined as the measure of heat transfer intensity. Apparently, according to Eq. (7), greater heat transfer intensity would yield a thicker molten pool at a prescribed time. In a similar manner, the dimensionless heat transfer intensity is defined as follows:

$Q_{f}=\frac{q_{0}^{\prime \prime} r_{0}}{\alpha \rho L}$.

Fig. 15a and b illustrate the effects of $Q_{f}$ on the molten pool shape. The maximum interfacial position of molten pool along $x$-axis $\left(X_{\max }\right)$ increases with increasing $Q_{f}$, a self-evident result. A reasonable expectation is to have $26 \%(\sqrt[3]{2}-1)$ increase in $X_{\max }$ when $Q_{f}$ is doubled. However, $X_{\max }$ increases only by $12 \%$ at an expense of two times of $Q_{f}$ at $t=12 \mathrm{~s}$.

As Fig. 12 illustrates, $Y_{\max }$ varies only slightly before $t=40 \mathrm{~s}$. Subsequently, $Y_{\max }$ increases with $Q_{f}$. The corresponding increase at $t=96 \mathrm{~s}$ is approximately of $20 \%$, even greater than that of $X_{\max }(12 \%)$. Fig. $15 \mathrm{c}$ depicts the magnification plot of the $Y_{\max }$ versus $t$ data, using $Q_{f}$ as a parameter. At short time internal, the dependence of $Q_{f}$ on $Y_{\max }$ could even reverse; that is, $Y_{\max }$ increases at a declining rate with increasing $Q_{f}$.

The higher $Q_{f}$ yields the early initiation of Marangoni flow during melting, thereby having more enhanced heat transfer at rim region, and the essential role of heat conduction of liquid close to the central regime. After the initial stage (exceeding $40 \mathrm{~s}$ in the present sample calculation) the action of Marangoni flow becomes effective over the entire pool. The thickness of molten pool hence increases with $t$ and $Q_{f}$.

\subsection{Effects of Marangoni number}

The mean temperature of bulk fluid is herein defined as the area mean over all finite elements during numerical calculations as follows: 


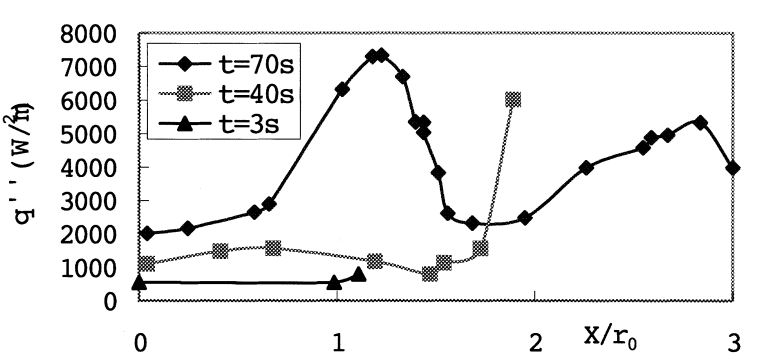

(a)

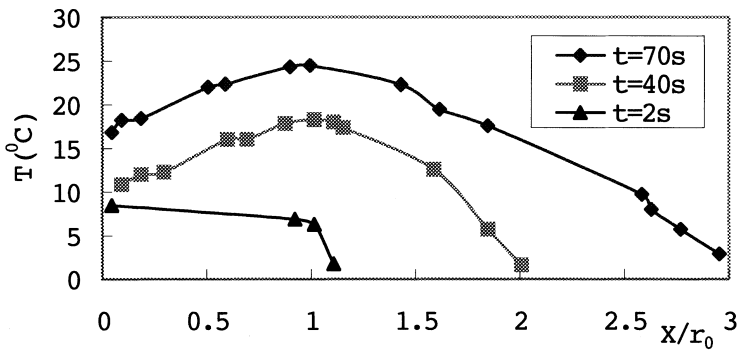

(b)

Fig. 13. (a) Heat flux at the solid-liquid interface for different time; (b) liquid temperature at free liquid surface for different time (pool I).

$T_{\text {mean }}=\frac{\int_{A} T \mathrm{~d} A}{\sum A_{i, j}}$.

Fig. 16 illustrates the evolutions of bulk mean temperatures considering pure heat conduction or a combined effect with the assistance of Marangoni flow. Notably, since the presence of Marangoni flow, the bulk liquid has been more readily mixed thereby yielding a lower bulk temperature when compared the case with pure heat conduction.

The Marangoni number denotes the ratio between surface tension driven heat transfer and the conduction heat transfer. To increase $M a$ represents enhanced Marangoni effects during melting.

Fig. 17 demonstrates the effects of $M a$ on the evolutions of bulk mean temperature. As expected, $M a$ has a negligible effect on the mean temperature at the initial stage, but reduces $T_{\text {mean }}$ at a long melting time.

\section{Conclusions}

Numerical investigations of solid-liquid phase changes require the initial molten pool for initiating the integration. Such an information is, however, still largely lacking in literature. This study investigated the role of initial molten pool on the melting process. The critical time for heat conduction mechanism to control the formation of initial molten pool is first analytically and theoretically estimated. A realistic formulation to the initial molten pool is proposed. Linear stability analysis was conducted for the critical film thickness beyond which Marangoni flow could be initiated. Three initial pools were employed in simulation. For initial pool having a melting time less than the critical time estimated herein, the subsequent numerical results are identical. Otherwise, erroneous numerical results could be generated in accordance with the adopted initial molten pool.

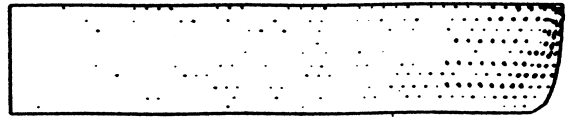

(a)

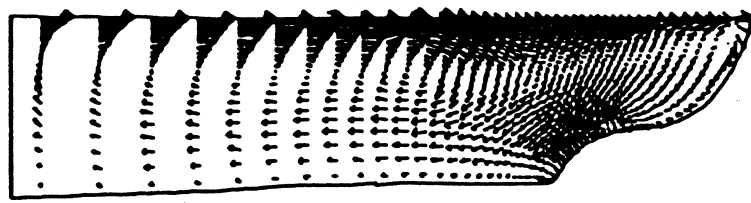

(b)

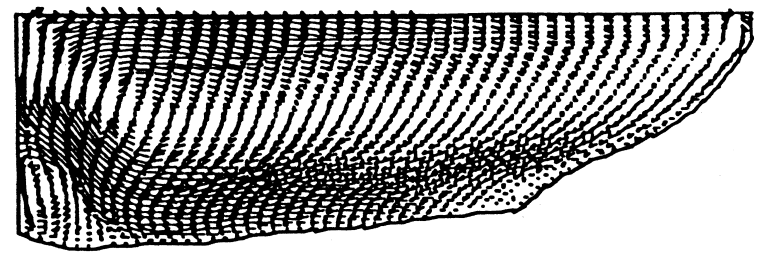

(c)

Fig. 14. (a) Velocity fileds at $t=2 \mathrm{~s}$ (pool I); (b) velocity fields at $t=40 \mathrm{~s}$ (pool I); (c) velocity fields at $t=70 \mathrm{~s}$ (pool I). 


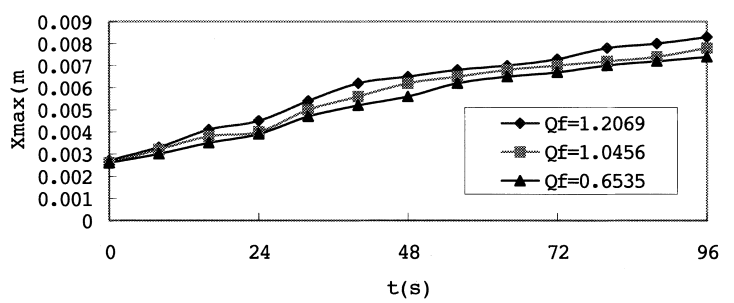

(a)

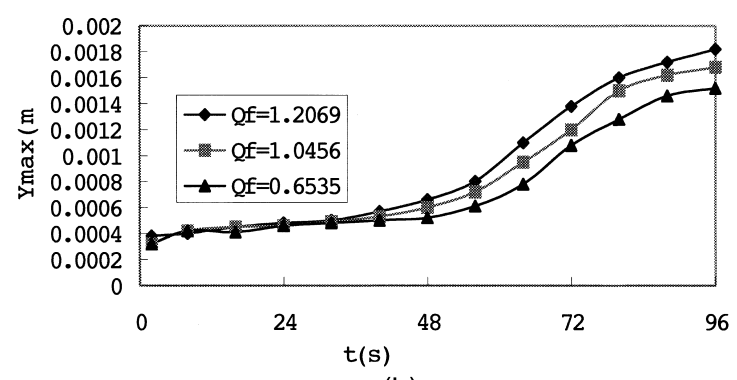

(b)

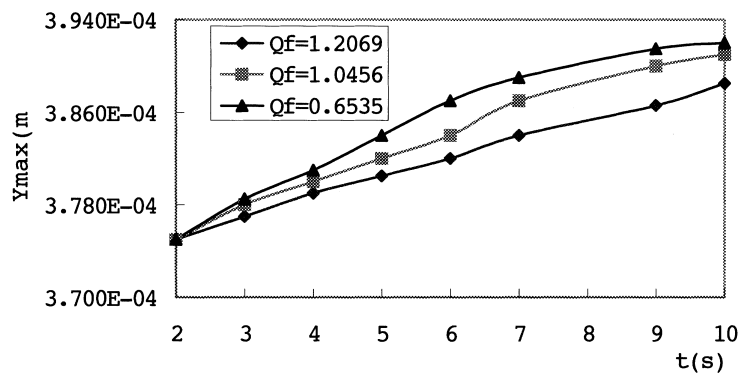

(c)

Fig. 15. (a) Effect of $Q_{f}$ on $X_{\max }$; (b) effect of $Q_{f}$ on $Y_{\max }$; (c) effect of $Q_{f}$ on $Y_{\max }$.

Initially, the molten pool mainly develops along the horizontal direction, with its pool thickness being almost invariant. The induced flow by Marangoni flow

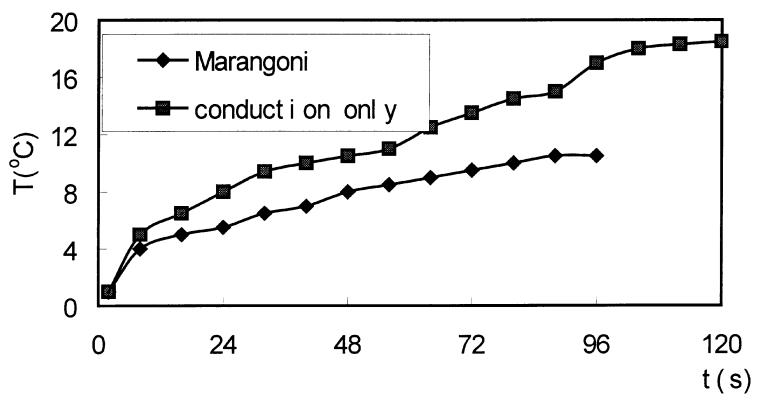

Fig. 16. Evolutions of bulk mean temperatures.

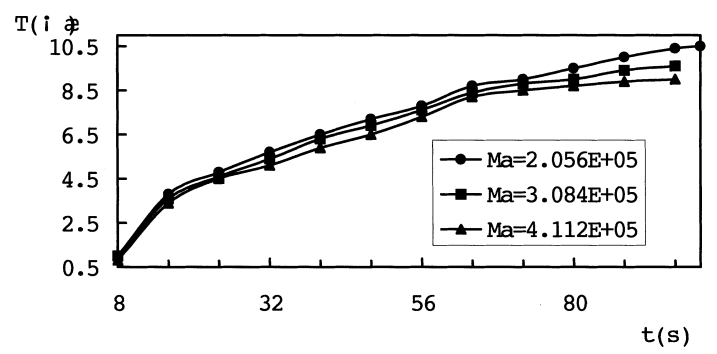

Fig. 17. Effect of $M a$ on the evolutions of bulk mean temperature. enhances the heat flux at the rim region. In the longterm dynamics, heat transfer enhancement regime moves to the symmetrical axis. The thickness of molten pool hence has been largely increased. Both the heat transfer intensity and the Marangoni number would markedly affect the temperature distributions, fluid flow fields, whence the shapes of molten pool at various stages.

\section{Acknowledgements}

This research is currently supported by the National Natural Science Foundation of China through contract No. 59625612.

\section{References}

[1] E.M. Sparrow, R.R. Schmidt, J.W. Ramsey, Analysis of melting in the presence of natural convection in the melted region, ASME J. Heat Transfer 99 (1977) 520526.

[2] N.W. Hale, R. Viskanta, Photographic observations of the liquid-solid interface motion during melting of a solid heated from an isothermal vertical wall, Lett. Heat Mass Transfer 5 (1978) 329.

[3] A.G. Bathlet, R. Viskanta, W. Leidenfrost, An experimental investigation of natural convection in the melted region around a heated horizontal cylinder, J. Fluid Mech. 90 (1979) 227-239. 
[4] L.S. Yao, F.F. Chen, Effects of natural convection in the melted region around a heated horizontal cylinder, ASME J. Heat Transfer 102 (1980) 667672.

[5] X.P. Lin, X.F. Peng, B.X. Wang, D.M. Christopher, Influence of Marangoni flow on the melting process, Porg. Nat. Sci. 7 (1997) 616-623.

[6] J. Srinivasan, B. Basu, A numerical study of thermocapillary flow in a rectangular cavity during laser melting, Int. J. Heat Mass Transfer 29 (1986) 563-573.

[7] T.L. Bergman, B.W. Webb, Simulation of pure metal melting with buoyancy and surface tension forces in the liquid phase, Int. J. Heat Mass Transfer 33 (1990) 139149.

[8] M. Akira, M. Sadanari, Pit formation in a polymer film by IR and UV laser irradiation, Proceedings of ICHMT Symposium, Yokohama, Japan, 1996.

[9] B. Basu, A.W. Date, Numerical study of steady state and transient laser melting problems - I. Characteristics of flow field and heat transfer, Int. J. Heat Mass Transfer 33 (1988) 1149-1163.

[10] B. Basu, A.W. Date, Numerical study of steady state and transient laser melting problems - II. Effects of process parameters, Int. J. Heat Mass Transfer 33 (1988) 1165-1175.

[11] T.R. Goodman, The heat-balance integral and its application to problem involving a change of phase, Trans. ASME 80 (1958) 335-342.

[12] J.R.A. Pearson, On convection cells induced by surface tension, J. Fluid Mech. 4 (1958) 489-500. 